\title{
BMJ Open Building a collaborative culture in cardiothoracic operating rooms: pre and postintervention study protocol for evaluation of the implementation of teamSTEPPS training and the impact on perceived psychological safety
}

Aaron Benjamin Dahl, ${ }^{1}$ Arbi Ben Abdallah, ${ }^{1}$ Hersh Maniar, ${ }^{2}$ Michael Simon Avidan, ${ }^{1}$ Mara L Bollini, ${ }^{1}$ George Alexander Patterson, ${ }^{2}$ Aaron Steinberg, ${ }^{1}$ Katie Scaggs, ${ }^{2}$ Brenda V Dribin, ${ }^{3}$ Clare H Ridley ${ }^{1}$

To cite: Dahl AB, Ben Abdallah $\mathrm{A}$, Maniar $\mathrm{H}$, et al. Building a collaborative culture in cardiothoracic operating rooms: pre and postintervention study protocol for evaluation of the implementation of teamSTEPPS training and the impact on perceived psychological safety. BMJ Open 2017;7:e017389. doi:10.1136/ bmjopen-2017-017389

- Prepublication history for this paper is available online. To view these files please visit the journal online (http://dx.doi. org/10.1136/bmjopen-2017017389).

Received 24 April 2017 Revised 4 August 2017 Accepted 11 August 2017

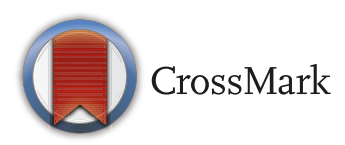

${ }^{1}$ Department of Anesthesiology, Washington University School of Medicine, Saint Louis, Missouri, USA

${ }^{2}$ Department of Cardiothoracic Surgery, Washington University School of Medicine, Saint Louis, Missouri, USA

${ }^{3}$ Dribin \& Associates, Saint Louis, Missouri, USA

Correspondence to Dr Aaron Benjamin Dahl; abdahl@wustl.edu, dahla54@gmail.com

\section{ABSTRACT}

Introduction The importance of effective communication, a key component of teamwork, is well recognised in the healthcare setting. Establishing a culture that encourages and empowers team members to speak openly in the cardiothoracic (CT) operating room (OR) is necessary to improve patient safety in this high-risk environment. Methods and analysis This study will take place at Barnes-Jewish Hospital, an academic hospital in affiliation with Washington University School of Medicine located in the USA. All team members participating in cardiac and thoracic $\mathrm{OR}$ cases during this 17 -month study period will be identified by the primary surgical staff attending on the OR schedule. TeamSTEPPS (Team Strategies and Tools to Enhance Performance and Patient Safety) training course will be taught to all CT OR staff. Before TeamSTEPPS training, staff will respond to a 39-item questionnaire that includes constructs from the Agency for Healthcare Research and Quality Hospital Survey on Patient Safety Culture, Edmondson's 'Measure of psychological safety' questionnaire, and questionnaires on turnover intentions, job satisfaction and 'burnout'. The questionnaires will be readministered at 6 and 12 months. The primary outcomes to be assessed include the perceived psychological safety of CT OR team members, the overall effect of TeamSTEPPS on burnout and job satisfaction, and observed turnover rate among the $\mathrm{OR}$ nurses. As secondary outcomes, we will be assessing self-reported rates of medical error and near misses in the ORs with a questionnaire at the end of each case.

Ethics and dissemination Ethics approval is not indicated as this project does not meet the federal definitions of research requiring the oversight of the Institutional Review Board (IRB). Patient health information (PHI) will not be generated during the implementation of this project. Results of the trial will be made accessible to the public when published in a peer-reviewed journal following the completion of the study.

\section{Strengths and limitations of this study}

- Strongly supported by leadership across the board, including the Chairmen of Surgery and Anaesthesiology, the Nursing Association, as well as Barnes-Jewish Hospital Perioperative Services.

- Clinicians in all teams who take care of cardiac and thoracic surgery patients on a daily basis will participate in our project. With such broad support and meaningful commitment, we are optimistic that this project will have a high likelihood to succeed in achieving its stated aims.

- This will be the first systematic effort to address teamwork and collaborative culture building within the perioperative community in the cardiothoracic operating rooms (OR), including the nursing, surgical and anaesthesiology staff.

- This programme will also be unusual in addressing the key metrics of psychological safety, job satisfaction and workforce stability.

- Some cases may be missed at night and on weekends when the research assistant is not in the $\mathrm{OR}$.

- This is an observational trial which does not provide data quite as robust and unbiased as does a randomised controlled trial.

\section{INTRODUCTION}

\section{Background and rationale}

The importance of effective communication, a key component of teamwork, is well recognised in the healthcare setting. ${ }^{12}$ Behaviour-related breakdowns in communication were identified as root causes of over $70 \%$ of adverse events according to a publication by the Joint Commission. ${ }^{3}$ In the patient safety literature, it has been widely recognised that team performance is crucial to providing safe patient care. 
In 2013, the American Heart Association (AHA) published a landmark scientific statement, 'Patient Safety in the Cardiac Operating Room: Human Factors and Teamwork.' The AHA guidelines recommend team training to improve leadership, communication and situational awareness in all cardiac operating rooms (ORs) that should involve all members of the cardiac operating team (class I recommendation, level of evidence B). ${ }^{4}$ To date, this guideline has not been implemented at Barnes-Jewish Hospital (BJH). As a leading hospital and academic medical centre, committed to the highest quality of care and patient outcomes, we must address this challenge proactively and expeditiously.

The OR is a high-stress, high-stakes environment. Patients undergoing cardiothoracic (CT) surgeries are among the most vulnerable at $\mathrm{BJH}$. There is a strong need in this setting to build a culture of respect and effective communication among OR team members in order to provide the best care. An audit by an organisational development consultant, commissioned by Washington University's Department of Surgery, revealed opportunities to develop teamwork skills and psychological safety within and between professional teams in the CT ORs. Psychological safety reflects the extent to which key protagonists, including surgeons, anaesthesiologists and nurses, create the safety climate necessary to prevent medical errors. A striking finding from this internal report was that only one-third of the CT surgeons met the cut-off requirement for acceptable levels of emotional intelligence, which affects the comfort that staff have in communicating their concerns in order to prevent adverse events. Overall, leaders in our ORs appeared to have deficiencies in conflict management skills and adaptability. This presented a pressing challenge for structured, collaborative culture building.

The cost of employee turnover to the hospital is significant. Nineteen nurses and surgical technicians (approximately half) left their positions in the CT ORs in 2015 for a variety of reasons. The cost of recruiting and training an OR nurse is more than $\$ 80000$. According to $\mathrm{BJH}$ internal data, the cost of turnover of nurses and technicians alone was estimated to be more than $\$ 1520$ 000. Measuring turnover intentions anonymously may be more sensitive than observed turnover with regard to job satisfaction, but turnover intention has the more negative impact on productivity and patients' outcomes. Employee burnout also has important implications ranging from decreased empathy with patients to major medical errors. ${ }^{5}$ According to a large cross-sectional survey published in the Annals of Surgery, mental quality of life had a large adverse relationship with reporting an error in the previous 3 months. ${ }^{6}$ Burnout is associated with higher turnover levels along with other factors such as hours worked.

Good teamwork starts with a set of learnt attitudes, behaviours and values. ${ }^{7}$ We propose to teach the TeamSTEPPS (Team Strategies and Tools to Enhance Performance and Patient Safety) training course to all CT OR clinicians, including surgeons, anaesthesiologists, perfusionists and nursing staff in order to improve teamwork, empathy and conflict resolution skills. This will enhance the culture, leading to greater job satisfaction and decreased turnover intentions, as well as boosting safety for our patients.

\section{Objectives}

1. Improve psychological safety in the CT ORs so that all team members will voice concerns regarding safety issues. Research focusing on healthcare providers' perceptions of teamwork demonstrated that staff members' perceptions of teamwork and attitudes towards safety-relevant team behaviour were related to the quality and safety of patient care. ${ }^{8}$ Perceptions of teamwork and leadership style are associated with staff well-being, which may impact clinicians' abilities to provide safe patient care. ${ }^{8}$ We hypothesise that the TeamSTEPPS modules on communication, leadership skills, situation monitoring and mutual support will improve psychological safety. Our goal with readministration of the questionnaire at 6 and 12 months is to reach $90 \%$ positive endorsement/ approval of all seven items in the psychological safety domain.

2. Decrease turnover intentions with the implementation of TeamSTEPPS training. We plan to measure turnover intentions as well as observed turnover, as staff might want to leave but are prevented from doing so by social or financial considerations. We hypothesise that turnover intentions will decrease with improved communication and perceived psychological safety in the CT ORs.

3. Decrease employee burnout with the hypothesis that employees working in conditions where open communication is encouraged and celebrated will feel more invested in and satisfied with their line of employment. We will assess employee burnout at baseline and at 6 and 12 months after TeamSTEPPS training. We hypothesise that employee burnout will decrease with improved communication and perceived psychological safety in the CT ORs.

4. Improve error reporting. The rates of error reporting are known to be low among staff. TeamSTEPPS training encourages participants to monitor their environment and speak up if a problem exists. We will look at error rates reported in the Quality Assessment Reporting Center (QARC) during the academic year before and after TeamSTEPPS training. Participants in the OR will also respond to a survey asking if a medical error or near miss occurred, and whether or not it was reported.

\section{METHODS}

\section{Study design}

This collaborative and multidisciplinary project will introduce TeamSTEPPS for the first time in the perioperative environment at Washington University and BJH. This intervention is a multifaceted approach to building a collaborative 
environment, decrease burnout and decrease job turnover. The TeamSTEPPS training programme, developed by the Department of Defense in conjunction with the Agency for Healthcare Research and Quality (AHRQ), reduces surgical morbidity and mortality. ${ }^{9}$ The training focuses on communication, leadership skills, situation monitoring, mutual support and conflict management skills. We have chosen these goals precisely because they address the deficiencies and challenges that were highlighted in the audit conducted by the Department of Surgery in 2014.

This is a preliminary cohort study using a convenience sample where all OR caregivers at BJH CT ORs will be asked to participate. All surveys will be confidential and anonymous. Participants' names will not be recorded, but individual characteristics of the OR team members will be requested in the survey for subgroup analyses.

Before TeamSTEPPS training, CT staff will respond to a 39-item questionnaire that includes constructs from the AHRQ Hospital Survey on Patient Safety Culture (HSOPSC), ${ }^{10}$ Edmondson's 'Measure of psychological safety' questionnaire, ${ }^{11}$ as well as questionnaires on turnover intentions, ${ }^{12}$ job satisfaction ${ }^{13}$ and 'burnout'. ${ }^{14}$ The additional specific subfacets of the AHRQ HSOPSC that we are measuring include: organisational learningcontinuous improvement, teamwork within units, communication openness and non-punitive response to errors. Due to concerns over survey length, it was not possible to include the entire HSOPSC. Instead, we selected a subset of constructs that were of greatest interest to the improvement work. We selected full measures (ie, all items that measure a given construct) to preserve the psychometric integrity of the survey measure.

The questionnaires will be readministered at 6 and 12 months after all members of CT staff have finished the TeamSTEPPS training (see table 1 and online supplementary appendix a).

In the preparation phase, all members of the CT ORs will receive an email informing them about the initiative, followed by two division-wide morning conferences that all $\mathrm{OR}$ members will be required to attend.

Eight members from the CT ORs (two surgeons, one anaesthesiologist, two nurses, one perfusionist, one anaesthesia tech and one Certified Registered Nurse Anaesthetist) chosen for their leadership skills will undergo the 2-day
'Train the Trainer' TeamSTEPPS programme at BJH taught by a certified TeamSTEPPS trainer who is contracted to teach the course. These eight people will comprise a core multidisciplinary group of skilled and vested champions who will teach the selected modules and promote the skills taught by the TeamSTEPPS programme. During the implementation phase, members of the CT staff will be broken down into groups and trained on the modules, 'Communication', 'Leading teams', 'Situation monitoring' and 'Mutual support' in 1-hour increments on 4 separate days at a time agreed upon by all divisions.

At the end of each case, all team members in the OR will be approached with a short survey to state the extent to which they agree or disagree with the following statements: (1) Our team communicated effectively during the case. (2) I felt comfortable asking questions and expressing concerns to other team members. (3) A medical error occurred during this case that nearly or did impact patient care. If the participant says that an event occurred, there is a follow-up question asking if the event was reported. If not, the respondent will be asked to provide the reason for not reporting the event from a drop-down menu. The options will be: 'No time; Someone else is reporting; Concern about anonymity; Don't know how; Lawsuit Concern; Embarrassed; Concern about judgment from others; The error did not affect the patient.' Team members will be asked to identify their role but there is an option to remain anonymous. This will allow for ongoing assessment of teamwork and psychological safety. For cases not occurring during regular business hours, the research coordinator will email all staff members involved the day following the case.

As a qualitative component to this study, we will be interviewing a random sample of staff of all experience levels about the TeamSTEPPS intervention at 6 and 12 months after the training. This information, whether it be positive or negative, will be used in improving the refresher TeamSTEPPS courses.

\section{Setting}

This study will take place at $\mathrm{BJH}$, an academic hospital in affiliation with Washington University School of Medicine located in the USA. This trial has been designed in accordance with the STROBE (Strengthening the Reporting of Observational Studies in Epidemiology) checklist. ${ }^{15}$

Table 1 Timeline of the planned interventions

$\begin{array}{ll}\text { July } 2016 & \text { Baseline questionnaires sent to CT staff. A research assistant will be hired and trained. } \\ \text { October 2016 } & \text { Master Training Course taken by eight individuals. } \\ \begin{array}{l}\text { 28 November 2016 to } 8 \\ \text { February 2017 }\end{array} & \begin{array}{l}\text { CT staff members receive lectures on 'Communication', 'Leading teams', 'Situation monitoring' and } \\ \text { 'Mutual support'. }\end{array} \\ \text { April } 2017 & \text { Review of preliminary data and refresher course on TeamSTEPPS concepts to all OR members } \\ \text { June } 2017 & \text { Questionnaires repeated at 6 months after intervention completed. } \\ \text { December 2017 } & \text { Questionnaires repeated at 12 months. Staff turnover assessed. } \\ \text { March 2018 } & \text { Final data analysis and dissemination of study findings }\end{array}$

CT, cardiothoracic; OR, operating room; TeamSTEPPS, Team Strategies and Tools to Enhance Performance and Patient Safety. 
All CT OR team members are required to attend TeamSTEPPS training. We will identify cardiac and thoracic cases by the attending surgeon's last name.

\section{Participants}

We will include all OR team members participating in cardiac and thoracic OR cases during this 17-month study period by screening the OR schedule based on the primary attending's last name. All identified cardiac and thoracic cases during work hours will be surveyed during business hours.

\section{Variables}

The primary outcome is $>90 \%$ positive endorsement approval for all seven psychological safety questions on the 39-item survey. We will also be looking at psychological safety and teamwork satisfaction as queried in the OR surveys. Burnout and turnover intentions will be assessed in the 39-item survey.

As a secondary outcome, we will be looking for improved reporting of medical errors and near misses in the ORs with the QARC reporting system.

\section{Bias}

This study is predisposed towards social desirability bias as there may be unrecognised pressure placed upon the staff and study to demonstrate improvement following our proposed intervention. To attempt to mitigate this, respondents to the surveys have the opportunity to remain anonymous if they so choose.

\section{Statistical methods}

We will create a database for this study using Research Electronic Data Capture (REDCap). REDCap uses browser-based software for managing research data. We will have access to REDCap through the Division of Biostatistics and Informatics at Washington University. REDCap servers are securely housed and managed by the Division of Biostatistics. All REDCap data transmission is encrypted following the Health Insurance Portability and Accountability Act (HIPAA) security guidelines. Patient safety in this study will be addressed with a comprehensive data safety and monitoring plan that complies with National Institutes of Health guidelines. We plan to produce detailed standard operating procedures for all members of the research team. We also plan to train all members of the research team to take proactive steps to minimise the risk for missing data.

A trained research assistant will be responsible for collecting the responses to the questions in the short survey on an iPad, which is directly linked to REDCap for an immediate download. Following this encounter, the data will not be encrypted; however, only research assistants, the principle investigator and coauthors will have access to the specific findings. If an OR member did not have a chance to respond, they will be sent a link to an anonymous survey requesting their participation.

At the end of each case, staff will rate their perceptions of teamwork and the environment of psychological safety on a 5-point Likert scale. Specifically, staff will respond to

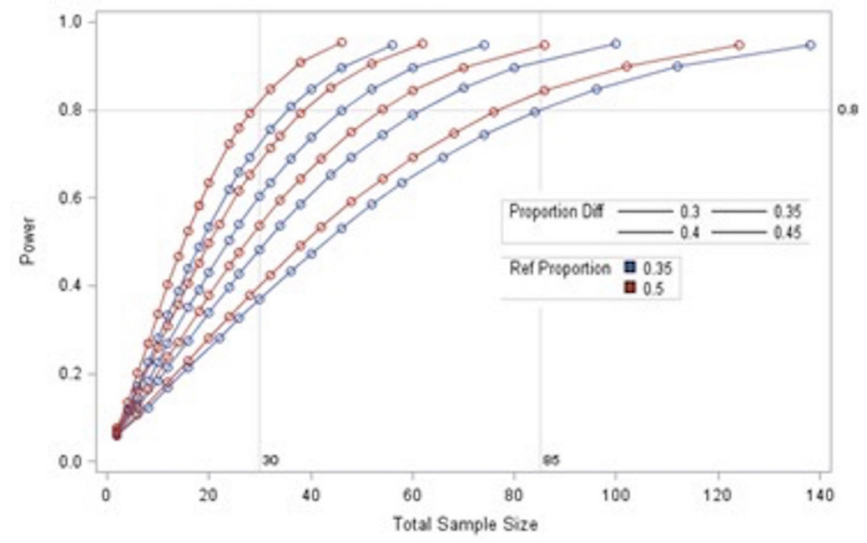

Figure 1 A graphical representation of the Pearson $\chi^{2}$ test of two proportions (each item was powered to detect a difference in proportions), assuming a minimum of $80 \%$ power and a two-sided $\alpha$ level of significance of 0.05 . It was determined that a maximum sample size of 85 participants is needed to achieve a $90 \%$ approval rate for all the scales in the psychological safety domain.

the questions, 'I am satisfied with the level of teamwork during this case' and 'I felt comfortable asking questions and expressing concerns to other team members.'

To test our main hypothesis that the TeamSTEPPS intervention in BJH CT ORs will improve each team member's interpersonal risk taking, learning behaviour and willingness to work together, we focused our power analysis on the psychological safety construct adopted from Edmondson. ${ }^{11}$ Psychological safety was chosen because it is a core domain for TeamSTEPPS intervention. It includes seven items: (1) 'If you make a mistake in this unit, it is often held against you', (2) 'Members of this unit are able to bring up problems and tough issues', (3) 'People in this unit sometimes reject others for being different', (4) 'It is safe to take a risk in this unit', (5) 'It is difficult to ask other members of this unit for help', (6) 'No one in this unit would deliberately act in a way that undermines my efforts' and (7) 'Working with members of this unit, my unique skills and talents are valued and utilized'. Based on our baseline survey conducted between July 2016 and December 2018 among all $(\mathrm{n}=110)$ clinicians in the CT ORs, the per cent of positive endorsement or approval for each of these seven items varied between $35 \%$ and $50 \%$. We defined our study aim to achieve $90 \%$ positive endorsement for all seven psychological safety questions on the 39-item survey following TeamSTEPPS training.

A statistical power analysis based on figure 1 was performed for all seven items in the psychological safety domain to define an overall sample size required for the entire study. The effective study sample was defined as the largest sample required by any of the seven psychological safety items. We powered each item to detect a difference in proportions using a Pearson $\chi^{2}$ test of two proportions, assuming a minimum of $80 \%$ power and a two-sided $\alpha$ level of significance of 0.05 . As shown in the graph below, a maximum sample size of 85 participants was needed to achieve a $90 \%$ approval rate for all the scales in the psychological safety domain. Furthermore, even if we experience an attrition 
rate of $20 \%$, the required sample $(n=102)$ will be still achievable $(n=110)$ to complete the study. Finally, we based our sample size calculation under conservative estimates which may be enhanced when using analytical techniques such as random regression.

The main survey used in this study comprised a 39-item questionnaire administered at baseline and at 6 and 12 months following TeamSTEPPS training. The questionnaire comprised 7 background and demographic questions as well as 32 items, covering 9 domains such as psychological safety ( 7 items), organisational learning (3 items), teamwork within hospital units (4 items), communication openness (3 items), non-punitive response to error (3 items), emotional exhaustion (4 items), job satisfaction (3 items), overall perception of safety (4 items) and an overall safety grade (1 item). Each domain item is scored on a 5-point Likert-type scale with responses ranging from $1=$ strongly disagree to 5 =strongly agree. Measures of each domain will be obtained at baseline, 6 months and 1 year after the four TeamSTEPPS training sessions provided to all CT OR clinicians. To evaluate each domain, each item as well as the average score across the domain items will be used to investigate the effects of the intervention. Descriptive statistics for all item responses and average domain score will be summarised at each time period and compared across time periods (baseline, 6 months and 1 year). We will apply Cochran-Mantel-Haenszel test or non-parametric analysis of variance (ANOVA) (Kruskal-Wallis) to compare differences in rating between OR team members as well as among group types at each study time period. We will also apply repeated measures ANOVA as well as analysis of covariance to assess the effects of the TeamSTEPPS intervention, using postintervention scores as the dependent variable and the level of the preintervention scores as well as the type of clinicians as covariates. A mixed effects model will also be used to assess the effect of the intervention while separating the subject (random) effects from the fixed effects. All these statistical analyses will be performed using SAS V.9.3 (SAS Institute).

\section{Ethics and dissemination}

The Human Research Protection Office at Washington University School of Medicine has reviewed this project and determined that it is a non-human subject research.

PHI will not be generated during the implementation of this project. Names of participants will not be associated with specific questionnaires. Participants have the option of identifying themselves or remaining anonymous when they respond to questionnaires. The principle investigator, coauthors and research assistants are the only participants who will have access to these data.

Results of the trial will be made accessible to the public when published in an accredited peer-reviewed journal following the completion of the study (see online supplementary appendix b).

\section{DISCUSSION}

\section{Strengths and limitations of this study}

The need for an intervention has been clearly identified and documented. This initiative is strongly supported by leadership across the board, including the Chairpersons of Surgery and Anaesthesiology, the Nursing Association, as well as BJH Perioperative Services. More importantly, we believe that those clinicians in all teams who take care of cardiac and thoracic surgery patients on a daily basis will enthusiastically embrace our project. With such broad support and meaningful commitment, we are optimistic that this project will have a high likelihood to succeed in achieving its stated aims. This will be the first systematic effort to address teamwork and collaborative culture building within the perioperative community in the CT ORs, including the nursing, surgical and anaesthesiology staff. This programme will also be unusual in addressing the key metrics of psychological safety, job satisfaction and workforce stability.

Contributors The manuscript was drafted by CHR, who is the Chief Investigator for Building a Collaborative Culture in Cardiothoracic Operating Rooms Trial. ABD is the first author with $A B A$ as the coauthor and statistician for this trial. HM, MSA, AS, MLB, GAP, KS and BVD are all coauthors. All authors contributed to the writing of this protocol and edited this manuscript for intellectual content.

Funding Funded by the Barnes-Jewish Hospital Foundation.

Competing interests None declared.

Provenance and peer review Not commissioned; externally peer reviewed.

Open Access This is an Open Access article distributed in accordance with the Creative Commons Attribution Non Commercial (CC BY-NC 4.0) license, which permits others to distribute, remix, adapt, build upon this work non-commercially, and license their derivative works on different terms, provided the original work is properly cited and the use is non-commercial. See: http://creativecommons.org/ licenses/by-nc/4.0/

(c) Article author(s) (or their employer(s) unless otherwise stated in the text of the article) 2017. All rights reserved. No commercial use is permitted unless otherwise expressly granted.

\section{REFERENCES}

1. Awad SS, Fagan SP, Bellows C, et al. Bridging the communication gap in the operating room with medical team training. Am J Surg 2005;190:770-4.

2. Gillespie BM, Chaboyer W, Murray P. Enhancing communication in surgery through team training interventions: a systematic literature review. Aorn J 2010;92:642-57.

3. JCAHO. Sentinel event statistics. Hospital authority Hong Kong: Annual report on sentinel events.2007.

4. Wahr JA, Prager RL, Abernathy JH, Jha I, et al. Patient safety in the cardiac operating room: human factors and teamwork: a scientific statement from the American Heart Association. Circulation 2013;128:1139-69.

5. Dyrbye L, Satele D, Collicott P, et al; Burnout and Medical Errors Among American Surgeons. , 2009:XXX(X), 1-6.

6. Shanafelt TD, Balch CM, Bechamps G, Russell T, et al. Burnout and medical errors among American surgeons. Ann Surg 2010;251:995-1000.

7. Gillespie BM, Chaboyer W, Longbottom P, et al. The impact of organisational and individual factors on team communication in surgery: a qualitative study. Int J Nurs Stud 2010;47:732-41.

8. Manser $\mathrm{T}$, . Teamwork and patient safety in dynamic domains of healthcare: a review of the literature. Acta Anaesthesiol Scand 2009;53:143-51.

9. Armour Forse R, Bramble JD, McQuillan R. Team training can improve operating room performance. Surgery 2011;150:771-8. 
10. Nieva VF, Sorra J. Safety culture assessment: a tool for improving patient safety in healthcare organizations. Qual Saf Health Care 2003;12 Suppl 2:17ii-23.

11. Edmondson A. Psychological safety and learning behavior in work teams. Adm Sci Q 1999;44:350.

12. Shore LM, Martin HJ. Job satisfaction and organizational commitment in relation to work performance and turnover intentions. Human Relations 1989;42:625-38.
13. Hackman JR, Oldham GR. Development of the job diagnostic survey $J$ Appl Psychol 1975;60:159-70.

14. Maslach C, Jackson SE. The measurement of experienced burnout. J Organ Behav 1981;2:99-113.

15. von Elm E, Altman DG, Egger M, et al. The strengthening the reporting of observational studies in epidemiology (strobe) statement: guidelines for reporting observational studies. PLoS Med 2007;4:e296. 